J. Dairy Sci. 98:6070-6084

http://dx.doi.org/10.3168/jds.2014-8914

(C) 2015, THE AUTHORS. Published by FASS and Elsevier Inc. on behalf

of the American Dairy Science Association ${ }^{\circledR}$. This is an open access article under

the CC BY-NC-ND license (http://creativecommons.org/licenses/by-nc-nd/3.0/).

\title{
Cost-benefit analysis of vaccination against Mycobacterium avium ssp. paratuberculosis in dairy cattle, given its cross-reactivity with tuberculosis tests
}

\author{
Huybert Groenendaal, ${ }^{* 1}$ Francisco J. Zagmutt, ${ }^{*}$ Elisabeth A. Patton, $†$ and Scott J. Wells $\ddagger$ \\ ${ }^{*}$ EpiX Analytics, Boulder, CO 80302 \\ †Wisconsin Department of Agriculture, Trade and Consumer Protection, Madison 53718 \\ $\ddagger$ College of Veterinary Medicine, University of Minnesota, St. Paul 55108
}

\begin{abstract}
Johne's disease (JD), or paratuberculosis, is a chronic enteric disease of ruminants, caused by infection with Mycobacterium avium ssp. paratuberculosis (MAP). Johne's disease causes considerable economic losses to the US dairy industry, estimated to be over $\$ 200$ million annually. Available control strategies include management measures to improve calf hygiene, testand-cull strategies, and vaccination. Although the first 2 strategies have shown to reduce the prevalence of MAP, they require dedicated and long-term efforts from dairy producers, with often relatively slow progress. As a result, uptake of both strategies has not been as wide as expected given the economic benefits especially of improved hygiene. Vaccination has also been found to reduce the prevalence and economic losses of JD, but most economic estimates have been based on simulation of hypothetical vaccines. In addition, if an animal is vaccinated, cross-reactivity between MAP antibodies and bovine tuberculosis (BTB) antigens may occur, decreasing the specificity of BTB tests. Therefore, MAP vaccination would cause additional indirect costs to the BTB surveillance and control program. The objective of the present study was to use data from a MAP vaccine trial together with an epidemiologic and economic model to estimate the direct on-farm benefits of MAP vaccination and to estimate the indirect costs of MAP vaccination due to the cross-reactivity with BTB tests. Direct economic benefits of MAP vaccination were estimated at $\$ 8.03$ (90\% predictive interval: $-\$ 25.97$ to $\$ 41.36)$ per adult animal per year, all accruing to the dairy producers. This estimate is likely an underestimation of the true direct benefits of MAP vaccination. In addition, indirect economic costs due to cross-reactivity
\end{abstract}

Received September 29, 2014.

Accepted April 22, 2015.

${ }^{1}$ Corresponding author: Huybert@epixanalytics.com were $\$ 2.14$ per adult animal per year, making MAP vaccination economically attractive. Only in regions or states with a high frequency of BTB testing (because of, for example, Mycobacterium bovis outbreaks in a wild deer population) and areas where typically small groups of animals are BTB tested would MAP vaccination not be economically attractive.

Key words: Johne's disease, vaccination, economic analysis, bovine tuberculosis program, cross-reactivity

\section{INTRODUCTION}

Johne's disease (JD), or paratuberculosis, is a chronic enteric disease of cattle and other ruminants infected by Mycobacterium avium ssp. paratuberculosis (MAP). In the dairy industry, JD is important due to the farm-level economic losses it causes (Ott et al., 1999). In addition, although not proven, there is concern about the potential causal role of MAP in Crohn's disease in humans [National Research Council (NRC) Committee on Diagnosis and Control of Johne's Disease, 2003]. Several measures are available to control the spread of MAP on dairy farms including test-andcull, management changes, and vaccination [National Research Council (NRC) Committee on Diagnosis and Control of Johne's Disease, 2003]. However, because of the low sensitivity of diagnostic tests for MAP, strategies based on test-and-cull alone are not effective in reducing its prevalence (Groenendaal et al., 2003; Dorshorst et al., 2006). In addition, the costs of test-andcull strategies tend to be higher than their economic benefits (Groenendaal et al., 2003; Kudahl et al., 2007). Improved calf management practices tend to be more effective in reducing the incidence of infection (Espejo et al., 2014) and within-herd MAP prevalence (Collins et al., 2010; Radia et al., 2013), and the farm-economic benefits of improving management practices have been estimated higher than their costs (Groenendaal et al., 2003; Dorshorst et al., 2006). 
A combination of test-and-cull strategies and management practices is often recommended to reduce the prevalence of MAP, while simultaneously making it economically attractive to the producer (Groenendaal et al., 2003; Dorshorst et al., 2006). The challenge with this combined strategy, however, is that it takes considerable resources and long-term efforts before seeing a benefit. Adoption of these control strategies by producers has therefore been limited.

Vaccination against MAP requires less effort by producers, and field studies that have evaluated MAP vaccination on MAP-infected herds have generally concluded that it effectively reduces fecal shedding of MAP (Knust et al., 2013) and the number of clinical JD cases, and that it can be cost-efficient at the herd level for high-prevalence herds (van Schaik et al., 1996). Simulation studies to evaluate the effectiveness of vaccination against MAP and its economic consequences have made similar conclusions but were often based on hypothetical ("potential") assumptions about the effectiveness of MAP vaccines (Groenendaal et al., 2003; Cho et al., 2012; Lu et al., 2013). Moreover, an important drawback of MAP vaccination is that it can interfere with the diagnosis of bovine tuberculosis (BTB) and therefore with BTB eradication and surveillance programs. Vaccination causes a greater percentage of false-positive BTB tests to occur in MAP-vaccinated animals (i.e., MAP vaccination lowers the specificity of the BTB tests), resulting in higher costs due to additional tuberculosis testing costs, and losses due to more herd quarantines. Dressler et al. (2010) examined the economic effects of this cross-reactivity and found that the benefits of vaccination exceed the costs of Johne's cross-reactivity. However, their study was based on an estimate of the benefits of MAP vaccination in herds with a very high MAP prevalence. In addition, they assumed a herd that is located within a geographic region with a tuberculosis outbreak attributed to surrounding endemically infected wild deer population.

To our knowledge, no empirical study has simultaneously considered the economics of MAP vaccination and its economic consequences on the BTB surveillance and eradication program. The objective of the current study was 2 -fold. First, the study used a previously developed empirically based simulation model of MAP vaccination on dairy herds (Zagmutt et al., 2012) to estimate the direct farm-economic consequences of MAP vaccination. Second, the study compared these direct farm-economic effects of MAP vaccination with its indirect economic effect on the BTB surveillance program. The paper provides decision support for producers and policy-makers regarding the economics of MAP vaccination based on empirical data.

\section{MATERIALS AND METHODS}

\section{Cost-Benefit Analysis and Overall Modeling Approach}

To achieve our objectives, 3 main steps were taken:

1. First, using a previously developed epidemiologic stochastic Markov Chain simulation model (Zagmutt et al., 2012) that was based on empirical data from a vaccination field trial (Knust et al., 2013), the direct farm-economic costs and benefits of MAP vaccination were estimated.

2. Second, the indirect costs of MAP vaccination to the BTB surveillance and eradication program due to cross-reactivity of MAP vaccinates with the caudal-fold tuberculin (CFT) and the comparative cervical tuberculin (CCT) tests were calculated.

3. Third, a cost-benefit analysis and a comprehensive sensitivity (break-even) analysis were performed to understand under what circumstances and assumptions MAP vaccination is economically attractive when taking into account its effects on the BTB program.

In all steps of the economic analysis, only the marginal costs and benefits due to MAP vaccination were considered. Table 1 provides an overview of the main costs and benefits taken into account in the cost-benefit analysis.

The default perspective of economic analysis is the society's perspective, disregarding which stakeholders accrue the different costs and benefits. However,

Table 1. Overview of main costs and benefits of Mycobacterium avium ssp. paratuberculosis (MAP) vaccination, how they are estimated, and payers or beneficiaries

\begin{tabular}{llll}
\hline Direct or indirect & Costs or benefit & Estimation approach/method & Payer or beneficiary \\
\hline Direct & Costs of vaccine & Calculated in the MAP simulation model & Producers \\
Direct & $\begin{array}{l}\text { Benefits due to the reduction of } \\
\text { Johne's disease losses }\end{array}$ & Calculated in the MAP simulation model & Producers \\
Indirect & $\begin{array}{l}\text { Costs to the bovine tuberculosis } \\
\text { surveillance program }\end{array}$ & Separate analysis & $\begin{array}{l}\text { Costs to producers and to state } \\
\text { or federal government }\end{array}$ \\
\hline
\end{tabular}


because not all costs and benefits accrue to the same stakeholders, an economic analysis was also performed separately, from both the producer's perspective and from the taxpayer's perspective.

\section{Direct Farm-Level Economic Effects of MAP Vaccination}

A previously developed Markov Chain model and its parameter estimates (Zagmutt et al., 2012) were used to estimate the within-herd epidemiological effects of MAP vaccination. In short, the model included 7 MAP-infection or disease states: (1) susceptible, (2) nonshedder adult, (3) latent, (4) low shedder, (5) heavy shedder, (6) clinical, and (7) culled. The main data set used (described by Knust et al., 2009, 2013) was from a 6-yr longitudinal data set of a controlled clinical trial on 3 MAP-infected Wisconsin (WI) dairy herds (A, B, C). Herd size ranged from 325 to 825 cows and, before participation in the trial, annual culling losses attributable to clinical JD within the 3 herds were about 6 to $8 \%$. For the first 10 -mo period of the trial, every other heifer calf born in each herd was administered a killed MAP vaccine, ensuring similar exposure for vaccinated and nonvaccinated cattle from a given herd. After this period, all heifer calves subsequently born within the 3 herds were vaccinated. During the study period, additions were made to the 3 herds. Despite recommendations to only purchase animals from tested sources, the cattle added to herds were from untested or unknown sources. The data set used in this study contained $5 \mathrm{yr}$ of survival and testing data for the 162 vaccinated and 145 control (i.e., nonvaccinated) heifers enrolled during the first 10 mo of the study. Fecal samples from all vaccinated and control animals were collected before each calving and at culling, independent of reason, over a period of $7 \mathrm{yr}$.

With the 6-yr longitudinal data set and using a latent-class Bayesian model, JD diagnostic test parameters were estimated, which were then used to simulate a longitudinal MAP infection trajectory for each vaccinated and control animal (Zagmutt et al., 2012; Espejo et al., 2015). The disease trajectories were subsequently used to estimate the joint uncertainty distributions of the transition probabilities of the stochastic Markov Chain model. The transition probabilities were then used to project the annual expected progression of MAP within the herd over 20 yr (Zagmutt et al., 2012). To determine the effect of MAP vaccination on the spread of MAP within the herd, the annual within-herd spread parameters (i.e., transition probabilities) were estimated separately for vaccinated and unvaccinated animals in the 3 herds. For an overall assessment of the effect of vaccination, the within-herd MAP spread parameters of the 3 herds were weighted equally. Given the industry-level perspective of the current study, only parameter uncertainty was considered and no randomness was included in the spread of MAP within individual herds.

Given the very low prevalence of BTB within the United States (i.e., the current surveillance efforts detect a $0.0002 \%$ BTB prevalence with $95 \%$ confidence; USDA-APHIS-VS, 2009), and because $>90 \%$ of US dairy operations have cattle infected with MAP (Lombard et al., 2013), the analysis' perspective was of a BTB-free and MAP-infected herd with a true MAP prevalence of $10 \%$ of shedding animals (low and heavy shedders and clinical animals). To show the effect of the within-herd prevalence at the start of vaccination, the model was also run using within-herd prevalences ranging from 5 to $35 \%$ with increments of $5 \%$ (based on Raizman et al., 2011).

The direct costs of MAP vaccination were calculated within the MAP infection simulation model by multiplying the costs of vaccination (assumed at $\$ 12 /$ heifer calf, based on discussions between author E. A. Patton and private practitioners in WI) by the number of new heifer calves each annual time-step within the MAP simulation model.

The direct net benefits of MAP vaccination were defined as the difference in losses between the situations with vaccination and without vaccination (i.e., the reduction of losses because of vaccination), also taking into account the costs of vaccination. Losses in either of the 2 situations were estimated by simulating the expected within-herd MAP infection dynamics over a 20-yr period and attributing different losses to the different MAP infection statuses. The direct benefits of MAP vaccination were then evaluated by comparing losses (and vaccination costs) in the 2 situations.

Table 2 shows the 3 types of direct losses due to MAP-infected animals that were included within the model: (1) The reduction in 305-d milk production of MAP-infected animals was based on the reduction in milk production described by Groenendaal and Wolf (2008). A milk price of $\$ 0.44 / \mathrm{kg}(\$ 20 / 100 \mathrm{lb}$.) was assumed (NASS, 2014). (2) The loss due to a lower slaughter value of animals with clinical JD was assumed at $\$ 353$ (Groenendaal and Wolf (2008), adjusted for inflation to 2014). (3) The third category of losses of JD included was caused by suboptimal (premature) culling of MAP-infected animals (Groenendaal et al., 2004). For each culled and infected animal, the loss due to suboptimal culling was set equal to the difference in retention pay-off value of the animal with and without the JD-related reduction in milk production. 
Table 2. Overview of direct losses due to Johne's disease (JD) included in the economic analysis

\begin{tabular}{lll}
\hline Description of loss & Economic effect & Source \\
\hline Milk production reduction & $\$ 96$ to $\$ 436 /$ animal per year, depending on disease status & Groenendaal and Wolf, 2008 \\
Lower slaughter value & $\$ 361$ per culled JD clinical animal & Groenendaal and Wolf, 2008, inflated to 2014 \\
Suboptimal culling & $\$ 192$ to $\$ 570$ per culled infected animal & Groenendaal et al., 2004, updated \\
\hline
\end{tabular}

The retention pay-off value of an animal is the expected profit from keeping the cow compared with immediate replacement and thus represents the loss due to suboptimal culling.

To calculate the total losses due to JD for the herd, the losses per animal were multiplied by the number of infected animals (in the 7 different infection statuses) and the number of culled infected animals as simulated by the MAP infection transmission model. The total annual net direct benefits due to MAP vaccination were subsequently calculated as the losses due to JD in the nonvaccination (NV) scenario minus the losses due to JD in the vaccination ( $\mathbf{V})$ scenario, minus the costs of MAP vaccination.

All total annual direct benefits were discounted at a 5\% annual discount rate to calculate the net present value (NPV). Net present value is a standard method for the financial appraisal of long-term projects and is used widely in economics (Brealey and Myers, 2007). Within an NPV, all net benefits over time (i.e., both costs and benefits) are discounted back to the start of the first year of the investment (in this situation, the first year of the 20-yr simulation) to account for the time-value of money and the riskiness of the investment decision. The assumed discount rate of $5 \%$ is higher than the risk-free rate but lower than discount rates generally used in other economic sectors. Finally, all NPV (calculated at the herd level over a 20 -yr period) were subsequently converted to their equivalent annual value per animal. Monte Carlo simulation using 10,000 iterations was performed using the ModelRisk software, version 5.1.0.3 (Vose Software, Ghent, Belgium).

\section{Indirect Effects of MAP Vaccination}

The indirect effects of MAP vaccination were defined as all costs due to additional false-positive diagnostic tests (because of cross-reactivity between MAP vaccination and BTB tests) within the current US BTB program (USDA-APHIS, 2004). Although accredited veterinarians and approved state or federal veterinarians have some discretion on which approved BTB diagnostic tests to use, we assumed the commonly used protocol, in which a full-herd CFT test was performed first, followed up by CCT test of all CFT responders and histopathology of all CCT reactors after euthaniz- ing the CCT-positive animals. No effect of MAP vaccination on the BTB slaughter surveillance was assumed because histology, PCR, and culture are used as the default test, which is not known to be affected by MAP vaccination.

The total annual expected indirect costs per animal due to MAP vaccination were calculated as the sum of the costs to producers and to the (state and federal) government:

Annual indirect costs/animal per year to producers $=$

$$
P_{\text {test }} \times\left[\begin{array}{l}
P_{\text {extra }}(\mathrm{CFT}+>0) \times \frac{\mathrm{C}_{\mathrm{QUAR}-\mathrm{PROD} 1}}{n} \\
+P_{\text {test }} \times P_{\text {extra }}(\mathrm{CCT}+>0) \times \frac{\mathrm{C}_{\mathrm{QUAR}-\mathrm{PROD} 2}}{n}
\end{array}\right]
$$

and

Annual indirect costs/animal per year to government $=$ $P_{\text {test }} \times \mathrm{CR}$ rate $_{\mathrm{CFT}} \times \mathrm{C}_{\mathrm{CFT} \text {-POS }}+P_{\text {test }} \times \mathrm{CR}$ rate $_{\mathrm{CFT}}$ $\times \mathrm{CR}$ rate $_{\mathrm{CCT}} \times \mathrm{C}_{\mathrm{CCT} \text {-POS }}+P_{\text {test }}$

$$
\times\left[\begin{array}{l}
P_{\text {extra }}(\mathrm{CFT}+>0) \times \frac{\mathrm{C}_{\mathrm{QUAR}-G O V T 1}}{n}+P_{\text {test }} \\
\times P_{\text {extra }}(\mathrm{CCT}+>0) \times \frac{\mathrm{C}_{\mathrm{QUAR}-\mathrm{GOVT} 2}}{n}
\end{array}\right],
$$

where $P_{\text {test }}=$ annual probability an animal is CFT tested within the BTB surveillance program; $P_{\text {extra }}(\mathrm{CFT}+$ $>0)=P(\mathrm{CFT}+>0 \mid \mathrm{V})-P(\mathrm{CFT}+>0 \mid \mathrm{NV})$; that is, the additional probability of at least $1 \mathrm{CFT}+$ due to vaccination; $P_{\text {extra }}(\mathrm{CCT}+>0)=P(\mathrm{CCT}+>0 \mid \mathrm{V})$ $-P(\mathrm{CCT}+>0 \mid \mathrm{NV})$; that is, the additional probability of at least $1 \mathrm{CCT}+$ due to vaccination; $n=$ number of animals CFT tested per testing round; CR rate $_{\mathrm{CFT}}=\left(\mathrm{Sp}_{\mathrm{CFT}}-\mathrm{Sp}_{\mathrm{CFT} \mid \mathrm{V}}\right)$, the CFT cross-reactivity rate; $\mathrm{CR}$ rate CCT $=\left(\mathrm{Sp}_{\mathrm{CCT}}-\mathrm{Sp}_{\mathrm{CCT} \mid \mathrm{V}}\right)$, the $\mathrm{CCT}$ crossreactivity rate; $\mathrm{Sp}_{\mathrm{CFT}}=$ specificity of CFT test; $\mathrm{Sp}_{\mathrm{CFT} \mid \mathrm{V}}$ = specificity of CFT test for MAP-vaccinated animal; $\mathrm{Sp}_{\mathrm{CCT}}=$ specificity of CCT test; $\mathrm{Sp}_{\mathrm{CCT} \mid \mathrm{V}}=$ specificity of CCT test for MAP-vaccinated animals; $\mathrm{C}_{\mathrm{CFT} \text {-POS }}=$ cost of an additional CCT test; $\mathrm{C}_{\mathrm{CCT} \text {-POS }}=$ costs of removal and histopathology, $\mathrm{PCR}$, and culture of an additional CCT-positive animal; $\mathrm{C}_{\mathrm{QUAR}-\mathrm{PROD} 1,2}=$ cost of 
Table 3. Overview of parameter values used to calculate the indirect costs of Mycobacterium avium ssp. paratuberculosis vaccination

\begin{tabular}{|c|c|c|c|}
\hline Parameter & Description $^{1}$ & Default & Source \\
\hline$n$ & Number of animals tested per herd per testing round & 300 & - \\
\hline $\mathrm{Sp}_{\mathrm{CFT} \mid \mathrm{V}}$ & Specificity of CFT test, vaccinated animal & $70 \%$ & Lima et al., 2012; Larsen et al., 1978 \\
\hline $\mathrm{Sp}_{\mathrm{CCT}}$ & Specificity of CCT test & $98.19 \%$ & Farnham et al., 2012 \\
\hline $\mathrm{Sp}_{\mathrm{CCT} / \mathrm{V}}$ & Specificity of CCT test, vaccinated animal & $90 \%$ & Lima et al., 2012; Larsen et al., 1978 \\
\hline $\mathrm{C}_{\text {QUAR-GOVT1,2 }}$ & $\begin{array}{l}\text { Government costs because of quarantine after either CFT+ } \\
\text { or CCT+ }\end{array}$ & $\$ 1,234$ & Dressler et al., 2010 \\
\hline $\mathrm{C}_{\text {QUAR-PROD1 }}$ & Producer costs because of quarantine after $\mathrm{CFT}+$ & $\$ 1,191$ & Dressler et al., 2010 \\
\hline Counar-prodi & Producer costs because of quarantine after CCT+ & $\$ 3,929$ & Dressler et al., 2010 \\
\hline
\end{tabular}

${ }^{1} \mathrm{CFT}=$ caudal-fold tuberculin; CCT $=$ comparative cervical tuberculin.

a quarantine period to producer after (1) at least one CFT-positive or (2) at least one CCT-positive animal; $\mathrm{C}_{\text {QUAR-GOVT1,2 }}=$ cost of a quarantine period to government after (1) at least one CFT-positive or (2) at least one CCT-positive animal. An overview of parameter values related to all indirect costs of MAP vaccination is shown in Table 3.

Within the US BTB program, only a fraction of all adult animals within the United States are tested each year. The probability that a random animal is BTB tested per year $\left(P_{\text {test }}\right)$ as part of the BTB surveillance program varies with (among others) the $M$. bovis status of the state and the location of the herd. Assuming that all animals within a region or state are equally likely to be tested, $P_{\text {test }}$ is the percentage of animals within a region or state that is BTB tested with a CFT test antemortem per year. In 2007, the percentage of cattle CFT tested within all US accredited-free states was $2.87 \%$; therefore, $P_{\text {test }}$ was set at $2.87 \%$ per animal per year (USDA-APHIS-VS, 2009). The $P_{\text {test }}$ within states ranged from $0.04 \%$ (Alaska) and $0.23 \%$ (Montana) to $34.39 \%$ (Rhode Island) and $34.52 \%$ (Arizona).

The specificity of the CCT test $\left(\mathrm{Sp}_{\mathrm{CCT}}\right)$ used in this study is conditional on a false-positive CFT test result (Farnham et al., 2012). The reported $\mathrm{Sp}_{\mathrm{CFT}}$ test varies greatly as illustrated by Farnham et al. (2012), who, in their meta-analysis, reported values ranging from 89.2 to $98.8 \%$ (weighted mean: $92.55 \%$ ). Also, MAP vaccination increases the number of false-positive results of the CFT (i.e., it reduces the specificity of the CFT test), as shown by Lima et al. (2012).

The costs per additional CFT-positive test $\left(\mathbf{C}_{\text {CFT-Pos }}\right)$ were assumed to be $\$ 4.41$ per animal based on Dressler et al. (2010). Regarding the costs due to an additional CCT positive test $\left(\mathbf{C}_{\mathrm{CCT}-\mathrm{POs}}\right)$, we assumed that CCT-positive animals would be slaughtered and histopathology performed (in practice, PCR and culture are also performed in certain situations). Indemnity depends on the value of the animal and was set at the maximum USDA indemnity value of $\$ 3,000$ per animal. Costs of histopathology to the state were assumed at $\$ 354$ per animal (USAHA, 2004; inflated to 2013).

The probability of at least one CFT+ animal, $P(\mathrm{CFT}+>0)$, is calculated as follows:

$$
P(\mathrm{CFT}+>0)=1-\mathrm{Sp}_{\mathrm{CFT}}^{n}
$$

where $\mathrm{Sp}_{\mathrm{CFT}}=$ specificity of the CFT test, and $n=$ number of animals tested per herd per testing round; $P(\mathrm{CFT}+>0)$ was calculated separately for vaccinated and nonvaccinated herds, and the difference of the 2 probabilities was equal to $P_{\text {extra }}(\mathrm{CFT}+>0)$. This extra probability of quarantine of a herd due to crossreactivity of MAP vaccination with the CFT test is then multiplied by the costs of quarantine due to a $\mathrm{CFT}+$ animal. The probability of at least one $\mathrm{CCT}+$ animal $[P(\mathrm{CCT}+>0 \mid \mathrm{V})$ and $P(\mathrm{CCT}+>0 \mid \mathrm{NV})$ for vaccinated and nonvaccinated herds, respectively] was calculated as follows:

$$
\begin{aligned}
& P(\mathrm{CCT}+>0)= \\
& \sum_{x=0 \rightarrow \infty}\left[\left(1-\operatorname{Sp}_{\mathrm{CCT}}\right)^{x}\left(\begin{array}{l}
n \\
x
\end{array}\right)\left(1-\operatorname{Sp}_{\mathrm{CFT}}\right)^{x}\left(\operatorname{Sp}_{\mathrm{CFT}}\right)^{(n-x)}\right],
\end{aligned}
$$

where $\mathrm{Sp}_{\mathrm{CCT}}=$ specificity of the CCT test, and $n=$ number of animals tested per herd per testing round, and all other terms are as defined previously; $\mathrm{P}(\mathrm{CCT}+>$ 0 ) was calculated separately for vaccinated and nonvaccinated herds, and the difference of the 2 probabilities was equal to $P_{\text {extra }}(\mathrm{CCT}+>0)$. This extra probability of quarantine of a herd due to cross-reactivity of MAP vaccination with the CCT test was then multiplied by the costs of quarantine due to a CCT + animal.

The total costs of a potential quarantine of a herd, during which no animals can leave the farm, due to 
MAP vaccination were assumed to be $\$ 2,425(\$ 1,234$ $+\$ 1,191)$ and $\$ 5,163(\$ 1,234+\$ 3,929)$ for the first $\mathrm{CFT}+$ and CCT+ animal, respectively.

\section{Cost-Benefit Break-Even Analysis}

The annual direct benefits of MAP vaccination per animal per year were compared with the indirect annual costs per animal per year due to cross-reactivity within the BTB surveillance program. Vaccination for MAP was considered economically attractive if the direct benefits of MAP vaccination were larger than the indirect costs.

Because the main parameters related to this costbenefit analysis are either uncertain or can vary between geographical areas within the United States, an extensive sensitivity analysis was performed to calculate the value of each of these input variables where the total indirect costs equal the total direct benefits. This sensitivity analysis therefore provides the combination of break-even parameters at which vaccination switched from economically attractive to economically unattractive and vice versa. The 7 parameters examined in this break-even analysis included (1) the direct net benefits of vaccination, (2) $P_{\text {test }}$, (3) $\operatorname{Sp}_{\mathrm{CFT} \mid \mathrm{V}}$, (4) $\mathrm{Sp}_{\mathrm{CCT} \mid \mathrm{V}}$, (5) $\mathrm{C}_{\text {QUAR-Prod1}}$, (5) $\mathrm{C}_{\text {QUAR-Prod2 }}$, and (6) $n$. Because the estimated annual direct benefits of MAP vaccination were based on disease-spread characteristics for only 3 WI dairy herds, all break-even analyses were conducted with 1 of the 2 varying parameters being the annual direct benefits of MAP vaccination. In addition to the direct benefits of MAP vaccination found within this study, the estimated direct benefits of MAP vaccination from van Schaik et al. (1996) and Groenendaal and Galligan (2003) are also displayed within each of the break-even analyses.

Related to $\mathrm{Sp}_{\mathrm{CFT} \mid \mathrm{V}}$ and $\mathrm{Sp}_{\mathrm{CCT} \mid \mathrm{V}}$, the actual specificity of either the CFT or CCT test is not important per se, but what is relevant is how the specificities are decreased because of MAP vaccination (i.e., the difference in specificity of either test for a MAP vaccinate and a MAP nonvaccinate). Therefore, in the break-even analysis, only the specificities of the CFT and CCT tests in MAP vaccinates were varied and not those in nonvaccinates. With regard to $\mathrm{C}_{\text {QUAR-PROD1 }}$ and $\mathrm{C}_{\text {QUAR-PROD2, }}$, both costs can vary greatly between states, production systems, and individual producers and were therefore examined within the break-even analysis.

In the default analysis, we assumed the commonly used protocol, in which a full-herd CFT test (herd of 300 adult animals) was performed. However, in certain states and situations smaller groups of animals are also CFT tested. Therefore, $n$ (number of animals tested per herd per testing round) was also varied in the break-even analysis.

Microsoft Excel's Solver, version 2010 (Microsoft Corp., Redmond, WA) was used to identify the break-even points, where direct benefits are equal to indirect costs, for all 7 parameters. The economic model that compares the direct economic benefits of MAP vaccination and the indirect economic costs is available at http://www.epixanalytics.com/ downloads/Paratuberculosis/TB-JDVaccinationCostBenefitAnalysis09-29-2014.xlsm.

\section{RESULTS}

\section{Direct Effects of MAP Vaccination}

Starting vaccination at a $10 \%$ within-herd prevalence, the expected forecasted within-herd prevalence without and with vaccination after $5 \mathrm{yr}$ was, respectively, $11.5 \%$ [95\% predicted interval (PI): 7.0 to $16.3 \%$ ] and $9.2 \%$ (95\% PI: 4.9 to 14.3\%; Figure 1).

Five and $10 \mathrm{yr}$ after start of the vaccination, the expected effect of vaccination (i.e., difference between $\mathrm{V}$ and NV scenarios) was a decrease in within-herd prevalence of $2.3 \%$ (95\% PI: -4.2 to $8.7 \%$ ) and $2.9 \%$ (95\% PI: -5.4 to $11.0 \%$ ), respectively.

When simulating individual herds, the expected number of infected animals during the first $5 \mathrm{yr}$ from the start of the vaccination decreased in the 3 herds by $2.5,21.9$, and $9.6 \%$ (overall $11.4 \%$ decrease) due to MAP vaccination (Table 4). The expected decrease of heavy shedders and clinical animals was greater on the 3 farms by $23.6,24.2$, and $3.4 \%$, respectively, and $17.0 \%$ overall.

Three years after starting vaccination at $10 \%$ withinherd prevalence, the expected annual net direct benefits per year became positive and increased until 5 to $7 \mathrm{yr}$ after the start of vaccination to approximately $\$ 12$ per animal per year (Figure 2). Expected net direct benefits of vaccination plateaued after about 5 to $7 \mathrm{yr}$, when the difference in within-herd prevalences of the $\mathrm{V}$ versus NV strategies stabilized (Figure 1). The effect of the MAP vaccination on direct MAP losses increased because it takes multiple years for vaccinated calves to because adult animals and thus for vaccination to have an effect on direct MAP losses.

Discounting back all net direct benefits of MAP vaccination, the expected annualized direct net benefits were estimated at $\$ 8.03$ per adult animal (90\% PI: $-\$ 25.97$ to $\$ 41.36)$. For herds A, B, and C, the direct benefits were estimated at $\$ 7.05(90 \%$ PI: $-\$ 31.63$ to $\$ 46.44)$ ), $\$ 13.23$ (90\% PI: $-\$ 14.13$ to $\$ 41.40)$, and $\$ 4.13$ (90\% PI: $-\$ 26.16$ to $\$ 33.71$ ), respectively (Table 5). 
Table 4. Mean (\%) and 90\% predicted interval (PI) range of the forecasted percentage change in the number of latent infected, low shedders, heavy shedders, and clinical animals due to vaccination in the first $5 \mathrm{yr}$ after starting Mycobacterium avium ssp. paratuberculosis vaccination

\begin{tabular}{lllccc}
\hline Herd & & Total infected & Latent infected & Low shedders & Heavy shedders and clinical \\
\hline A & Mean & -2.5 & -5.0 & 13.8 & -23.6 \\
& Range & -22.1 to 19 & -27.3 to 19 & -18 to 48.3 & -43.4 to -2.7 \\
B & Mean & -21.9 & -19.1 & -23.9 & -24.2 \\
& Range & -36.2 to -6.9 & -37.6 to 0.6 & -40.9 to -6.1 & -43.1 to -6 \\
C & Mean & -9.6 & -9.6 & -30.7 to 15.6 & -3.4 \\
& Range & -26.3 to 8.9 & -29.1 to 13.5 & -6.2 & -17.0 \\
& Mean & -11.4 & -11.4 & -33.9 to 27.3 & -40.7 to 10.2 \\
\hline
\end{tabular}

Herd B had the highest forecasted reduction in total infected animals and highest expected benefits. Herd C's reduction in losses was forecasted to be relatively limited, mostly because of a limited decrease in the number of high shedders and animals with clinical JD (i.e., the animals that cause the highest losses).

The expected direct benefits of MAP vaccination per animal per year increase when the prevalence at the start of the vaccination program is higher (Figure 3). Expected direct benefits of MAP vaccination increase from $\$ 7.00$ to $\$ 10.93$ per animal and year with an increase of the start prevalence of MAP shedders from 5 to $25 \%$.

\section{Indirect Effects of MAP Vaccination}

Two important parameters when calculating the indirect costs of MAP vaccination are the additional probabilities (due to MAP vaccination) of a quarantine due to a $\mathrm{CFT}+$ or $\mathrm{CCT}+$ animal, $P_{\text {extra }}(\mathrm{CFT}+>0)$ and $P_{\text {extra }}(\mathrm{CCT}+>0)$, respectively. Both parameters and $P(\mathrm{CCT}+>0)$ and $P(\mathrm{CFT}+>0)$ for vaccinated and nonvaccinated animals are shown in Figure 4 in relation to the number of animals that are CFT tested $(n)$ within a herd; CCT testing is conditional on CFT testing (i.e., when finding a CFT+ animal).

Figure 4 shows that when CFT testing groups of animals between 1 and 30, the difference between $\mathrm{P}(\mathrm{CFT}+$ $>0 \mid \mathrm{NV})$ and $\mathrm{P}(\mathrm{CFT}+>0 \mid \mathrm{V})$ ranges between 10 and $50 \%$, whereas with groups of 30 animals and larger, the difference quickly reaches $0 \%$. Therefore, if CFT tests within the BTB are typically performed on larger groups of animals, MAP vaccination could have no or very little effect on the probability of at least one falsepositive CFT test, whereas with smaller group sizes the effect can be considerable.

Furthermore, the difference between $\mathrm{P}(\mathrm{CCT}+>$ $0 \mid \mathrm{NV})$ and $P(\mathrm{CCT}+>0 \mid \mathrm{V})$ does reach a maximum

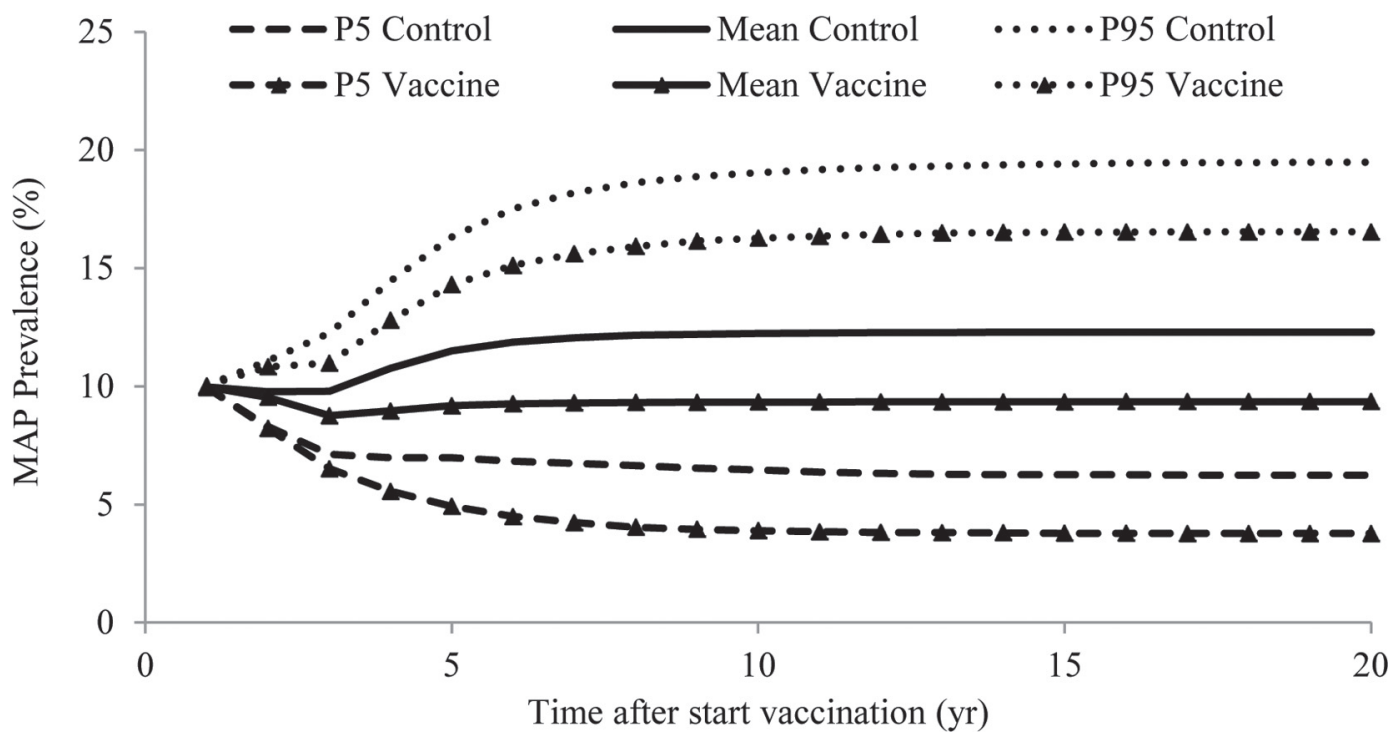

Figure 1. Within-herd prevalence of Mycobacterium avium ssp. paratuberculosis (MAP)-shedding animals (i.e., low shedders, heavy shedders, and clinical animals in the model) starting at $10 \%$ within-herd prevalence without vaccination (control) and with vaccination (vaccine). $\mathrm{P} 5$ and P95 $=5$ th and 95th percentiles, respectively. 


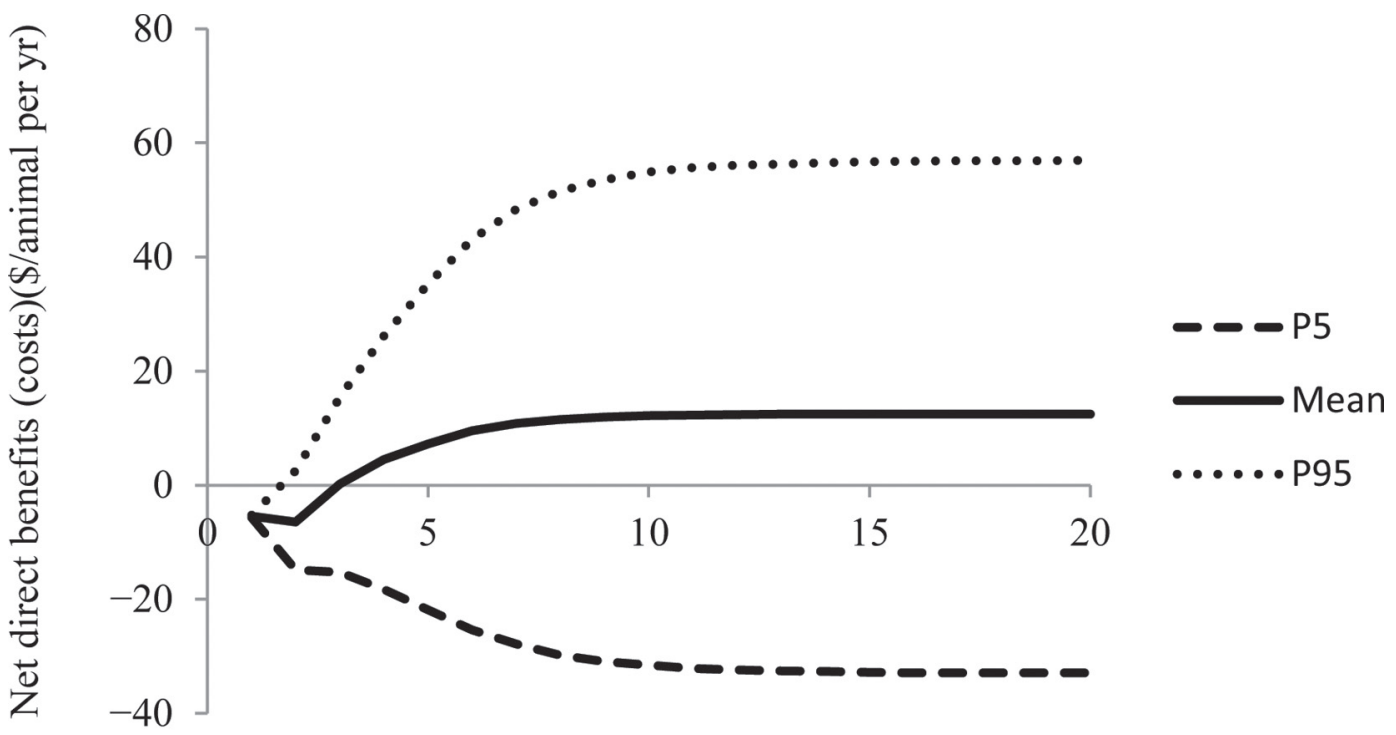

Time after start vaccination $(\mathrm{yr})$

Figure 2. Mean annual net direct benefits of Mycobacterium avium ssp. paratuberculosis (MAP) vaccination, including $90 \%$ predictive interval (PI) over 20-yr period, assuming $10 \%$ prevalence at start of vaccination. P5 and P95 = 5th and 95th percentiles, respectively.

when CFT testing groups of around 100 animals at a time, and the difference continues to be large even with much larger group sizes. Therefore, in contrast to $P_{\text {extra }}(\mathrm{CFT}+>0)$, across a wide range of group sizes, MAP vaccination considerably increases $P_{\text {extra }}(\mathrm{CCT}+$ $>0$ ), the probability of at least one CCT-positive animal.

Using the default values from Table 3, the expected indirect costs of MAP vaccination on the BTB program were calculated at $\$ 0.25$ at the herd level and $\$ 1.89$ at the government level, for a total of $\$ 2.14$ per animal per year. These indirect costs are lower than the direct benefits of MAP vaccination, indicating that

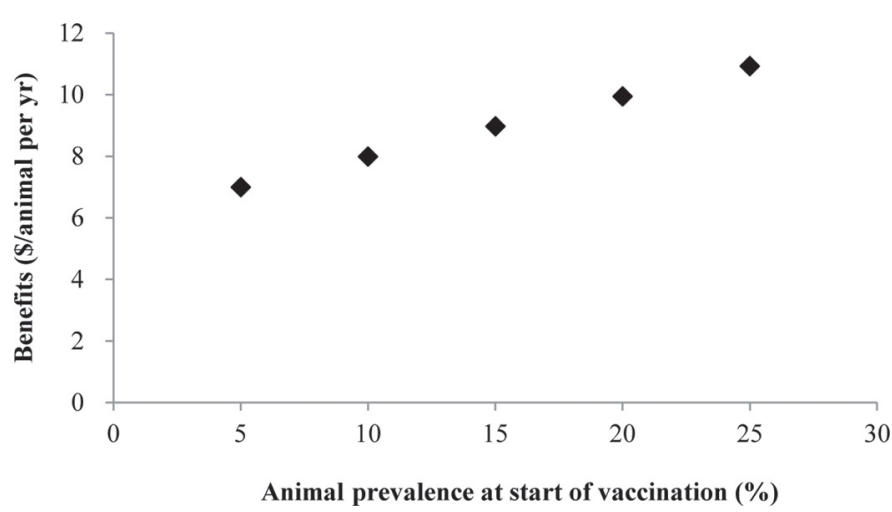

Figure 3. Expected net direct benefits of Mycobacterium avium ssp. paratuberculosis (MAP) vaccination per animal per year, assuming different within-herd animal prevalence levels of shedders at start of vaccination.
MAP vaccination from an overall societal perspective is economically attractive. Taking into account different economic perspectives, at the herd level, the expected net benefits are $\$ 7.77(\$ 8.02-0.25)$ per animal per year, whereas at the government level, the net benefits are $-\$ 1.89$ per animal per year.

\section{Cost-Benefit Break-Even Analysis}

The estimated annual direct benefits of MAP vaccination and the proportion of animals CFT tested per year $\left(P_{\text {test }}\right)$ both have an effect on the overall economic attractiveness of MAP vaccination (Figure 5A). Figure $5 \mathrm{~A}$ shows that vaccination is not economically attractive for herds in regions or states in which $P_{\text {test }}$ is high, because of a higher probability, for example, of a tuberculosis outbreak. It also shows that if the expected net direct benefits of MAP vaccination were higher than estimated in the current study, vaccination would also be economically attractive in areas with a higher $P_{\text {test }}$.

Table 5. Mean, 5th percentile (P5) and 95th percentile (P95) of direct net benefits of vaccination per adult animal per year, starting at a $10 \%$ within-herd Mycobacterium avium ssp. paratuberculosis prevalence of shedders

\begin{tabular}{lrcc}
\hline Herd & Mean & P5 & P95 \\
\hline A & $\$ 7.05$ & $-\$ 31.63$ & $\$ 46.44$ \\
B & $\$ 13.23$ & $-\$ 14.13$ & $\$ 41.40$ \\
C & $\$ 4.13$ & $-\$ 26.16$ & $\$ 33.71$ \\
Overall & $\$ 8.03$ & $-\$ 25.97$ & $\$ 41.36$ \\
\hline
\end{tabular}




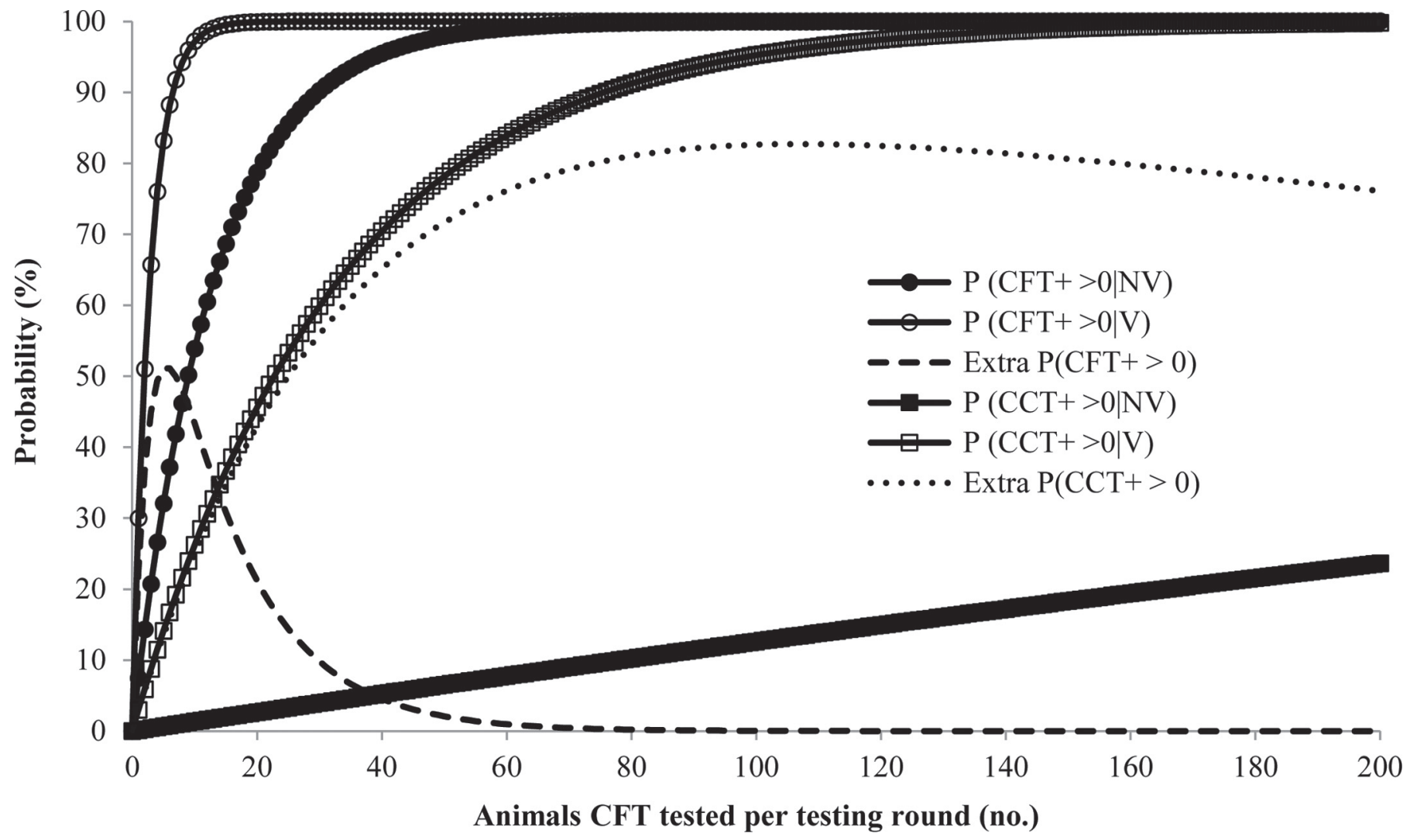

Figure 4. Probability of at least one false-positive caudal-fold tuberculin (CFT) $[P(\mathrm{CFT}+>0)]$ or comparative cervical tuberculin $(\mathrm{CCT})$ $[P(\mathrm{CCT}+>0)]$ test when testing a vaccinated $(\mathrm{V})$ or nonvaccinated $(\mathrm{NV})$ group of animals. Extra $P$ is the difference between the probability for a vaccinated and nonvaccinated group.

Second, the estimated annual direct benefits of MAP vaccination and the specificity of the CFT test for MAP vaccinates were varied (while keeping all other parameter at their default values, see Table 3). Figure $5 \mathrm{~B}$ shows that even if the specificity of the CFT for MAP vaccinates were only $50 \%$ (versus the assumed specificity of MAP nonvaccinates of $92.55 \%$ ), vaccination would still be economically attractive at current tuberculosis testing rates.

Third, the break-even analysis with varying annual direct benefits of MAP vaccination and specificity of the CCT test for MAP vaccinates shows that when the specificity of the CCT for vaccination is $70 \%$ or less and annual direct benefits of MAP vaccination are around $\$ 7 /$ animal, vaccination becomes economically unattractive (Figure 5C). Under the default 90\% specificity of the CCT test for MAP vaccinates, direct MAP vaccination benefits could be as low as $\$ 2$ /animal per year to get to the break-even point where direct benefits become equal to indirect costs.

The break-even analysis on the annual direct benefits of MAP vaccination and costs to the producers of a farm standstill because of a CFT-positive animal
$\left(\mathrm{C}_{\mathrm{QUAR}-\mathrm{PROD} 1}\right)$ shows that in the default situation whatever $\mathrm{C}_{\text {QUAR-PROD1}}$, MAP vaccination direct benefits exceed indirect costs. The reason for this is that with CFT testing of 300 nonvaccinates, the probability of a standstill because of at least one CFT-positive animal $[P(\mathrm{CFT}+>0 \mid \mathrm{NV}]$ would already be close to $100 \%$ (see Figure 4); therefore, vaccination could not further increase this probability, reducing to a negligible level the marginal effect of MAP vaccination on quarantines because of a CFT-positive animal. Therefore, an alternative break-even analysis was performed on the annual direct benefits of MAP vaccination and costs to the producers of a farm standstill because of a CFTpositive animal $\left(\mathrm{C}_{\mathrm{QUAR}-\mathrm{PROD} 1}\right)$, but with $n=10$ to represent situations or states where animals are typically CFT tested in smaller groups (Figure 5D). Figure 5D shows that using MAP vaccination direct benefits from the current studies (\$8.02/animal per year) and with $n=10$, overall vaccination is economically marginally unattractive. With slightly higher direct MAP vaccination benefits, lower quarantine costs (or when $n>$ 11, see Figure 5F), vaccination direct benefits exceed vaccination indirect costs. 
A)

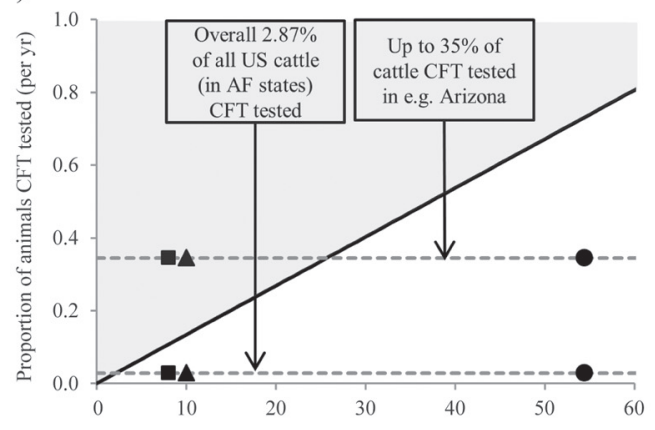

C)

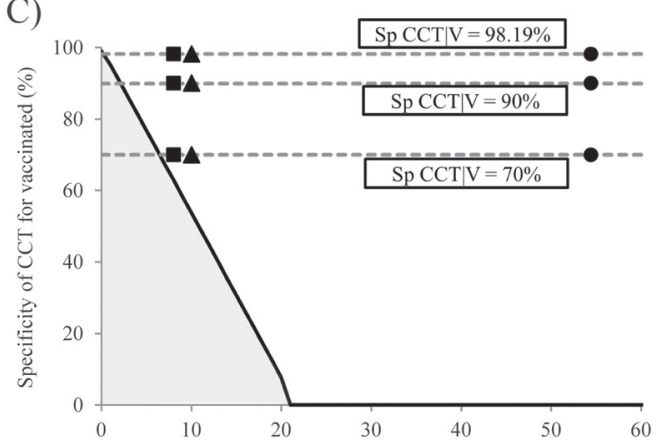

E)

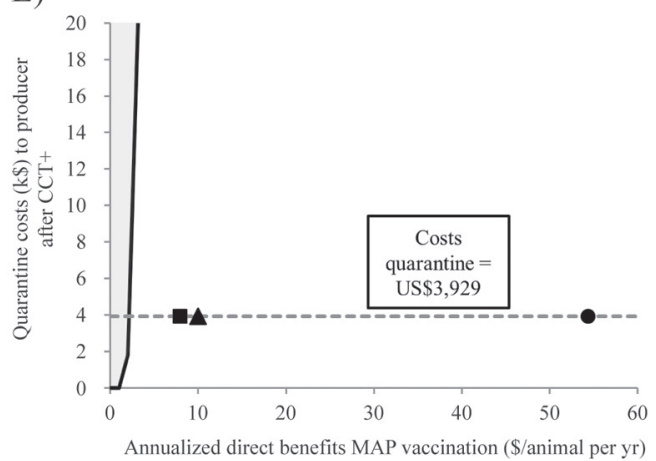

B)

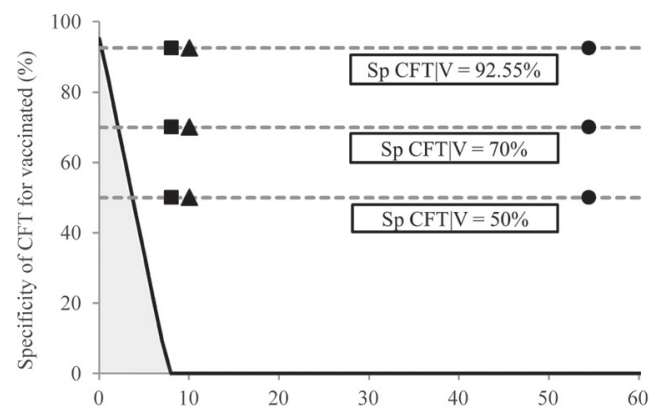

D)

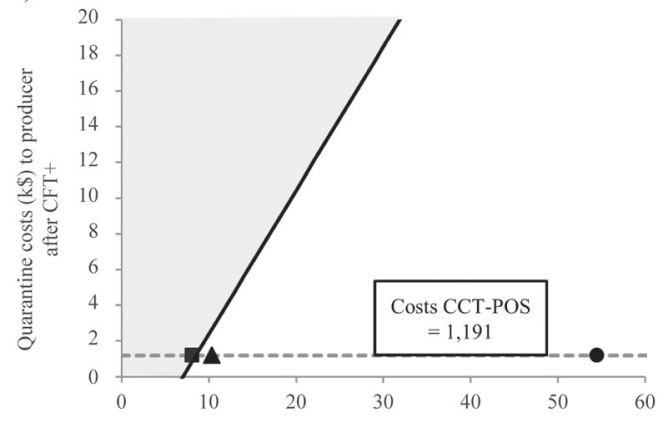

F)

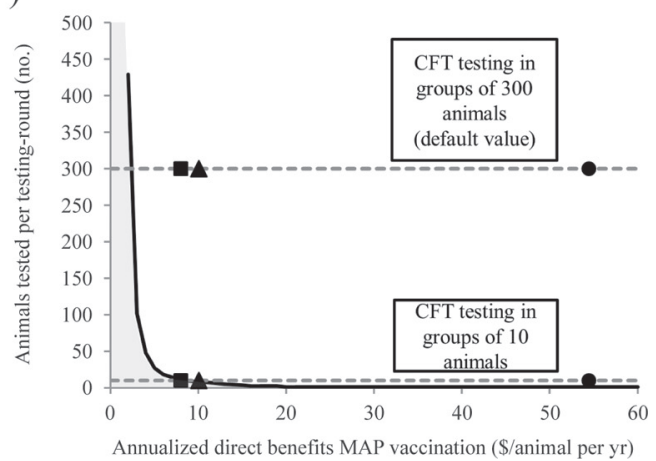

Figure 5. Break-even analysis of Mycobacterium avium ssp. paratuberculosis (MAP) vaccination with varying annual direct benefits of vaccination per animal, and varying (A) proportions of animals caudal-fold tuberculin (CFT) tested per year $\left(P_{\text {test }}\right)$, (B) specificity of CFT test for MAP-vaccinates $\left(\mathrm{Sp}_{\mathrm{CFT} \mid \mathrm{V}}\right),(\mathrm{C})$ specificity of comparative cervical tuberculin $(\mathrm{CCT})$ test for MAP-vaccinates (Sp $\left.\mathrm{CCCT}_{\mathrm{V}}\right)$, (D) costs to the producers of a farm standstill because of a CFT-positive animal ( $\mathrm{C}_{\text {QUAR-PROD1 }}$ ) with $n=10$, (E) costs to the producers of a farm standstill because of a CCT-positive animal $\left(\mathrm{C}_{\text {QUAR-PROD2 }}\right)$, and $(\mathrm{F})$ number of animals CFT tested per testing-round $(n)$. Any point in the gray area represents situations in which MAP vaccination is not economically attractive, whereas any point in the white area represents situations in which the benefits of vaccination are higher than the costs. Squares ( $\boldsymbol{\square})$ represent direct MAP vaccination benefits estimates from the current study, triangles $(\boldsymbol{\Delta})$ represent direct benefits from Groenendaal and Galligan (2003), and circles ( ) represent direct benefits from van Schaik et al. (1996), inflated to 2014 as per Dressler at al. (2010). AF = accredited-free.

The break-even analysis on the annual direct benefits of MAP vaccination and costs to the producers of a farm standstill because of a CCT-positive animal $\left(\mathrm{C}_{\mathrm{QUAR}-\mathrm{PROD} 2}\right)$ shows that even if $\mathrm{C}_{\mathrm{QUAR}-\mathrm{PROD} 2}$ is well over $\$ 3,929$, vaccination is economically attractive (Figure 5E).
Finally, based on the break-even analysis with varying annual direct benefits of MAP vaccination and varying number of animals CFT tested per testing round, MAP vaccination becomes economically indifferent when animals are CFT tested in groups of 11 or smaller (Figure 5F). 


\section{DISCUSSION}

\section{Direct Benefits of MAP Vaccination}

The present economic analysis shows that the expected direct benefits of MAP vaccination are positive, primarily as a result of vaccination reducing the frequency of heavy shedders and clinical animals within vaccinated herds. Expected net direct benefits per animal per year were $\$ 8.02$ for a herd with an initial $10 \%$ prevalence of MAP-shedding animals. The results also show that the effect of MAP vaccination on the overall prevalence and the prevalence of latent infected and low shedder animals is fairly small, whereas the effect on the number of heavy shedders and clinical animals is larger, which is consistent with earlier studies (Groenendaal and Galligan, 2003; Knust et al., 2013; Tewari et al., 2014).

Given the limited disease transmission data available from the $3 \mathrm{WI}$ dairy herds, there is considerable uncertainty (90\% PI from $-\$ 25.97$ to $\$ 41.36 /$ animal per year) regarding the net direct benefits of MAP vaccination presented in this study. Collecting additional data could reduce this parameter uncertainty, and therefore can provide decision-makers with greater confidence in their optimal decisions. A value-of-information analysis, similar to those performed in human health cost-effectiveness analysis, could help determine the value to decision-makers of collecting such additional information (Briggs et al., 2012).

When interpreting the results of the direct benefits of MAP vaccination, the following limitations and assumptions are important to consider. First, the disease-spread parameters of the epidemiological model were estimated based on only 3 WI dairy herds, and therefore the epidemiologic and economic predictions and results may not be applicable to other types of US dairies. Second, the disease-spread parameters (e.g., transition probabilities) of the stochastic Markov Chain model were estimated separately for vaccinated and the nonvaccinated animals within the same herds. The study design (Knust et al., 2013), where vaccinates and controls were systematically allocated within a farm, minimized the effect of differences in management practices between animals of the same herd. However, Knust et al. (2013) noted that farmers were not blind to the status of animals within their herd, which may have influenced some of the practices applied to vaccinated versus nonvaccinated animals. Then, as fewer vaccinated animals shed and did so at lower levels compared with the controls, vaccination of half of the herd likely had an effect beyond the protection of the vaccinated animals themselves and reduced the number of infections between nonvaccinated control animals in the study. In addition, before the start of the study, all cows were tested with an ELISA and in 2 of the 3 herds only heifer calves from ELISA-negative dams were retained in the herd during the duration of the study. Because the current study results depend on the difference between disease spread in controls and vaccinated animals, the results presented in the current paper therefore likely represent an underestimation of the true effect of vaccination that would result from the vaccination of all animals within a herd. On the other hand, the analysis by Knust et al. (2013) did not separate the effect of vaccination from the effect of management practices, which also help in limiting disease spread and contribute to the apparent effect of vaccination. In the study by Tewari et al. (2014), MAP vaccination decreased the prevalence and shedding, even in a herd that implemented virtually no change in preventive practices, supporting the conclusion that vaccination by itself has an effect on prevalence and shedding, but prevalence may be further decreased by implementation of good management practices. Third, the disease-spread model developed by Zagmutt et al. (2012) and used in this study was a Markov Chain model and thus assumed that infection probability did not decrease as the number of shedding animals decreased over time. Consequently, both the reduction of within-herd JD prevalence due to MAP vaccination and its economic effects are likely underestimations of the true direct benefits of vaccination. Two sensitivity analyses were performed, in which the MAP infection probability decreased an additional 50 and $100 \%$, respectively, over the simulated 20 -yr timeframe (i.e., this was a reduction beyond the one-time reduction seen during the $6 \mathrm{yr}$ of the MAP vaccination field trial on the WI dairy herds). Net expected direct benefits of MAP vaccination in both situations increased to $\$ 17.47$ and $\$ 28.90$ /animal per year, respectively (results not shown).

The expected net direct benefits found in this study were generally lower than in previous studies (Figure 6). van Schaik et al. (1996) reported net direct benefits of $\$ 54.42$ and $\$ 29.14$ per cow for herds with 11 and $5 \%$ of cows showing clinical JD at the start of vaccination, respectively (1996 estimates inflated to 2014 according to Dressler et al., 2010). Both situations imply a very high overall MAP prevalence and therefore, their estimates of the economic benefits of MAP infections are not representative of the typical MAP prevalence in a US herd. In addition, van Schaik et al. (1996) attributed the full reduction of the losses to vaccination, whereas some of the effects may have been due to improved hygiene management. On the farms used for disease-spread data 


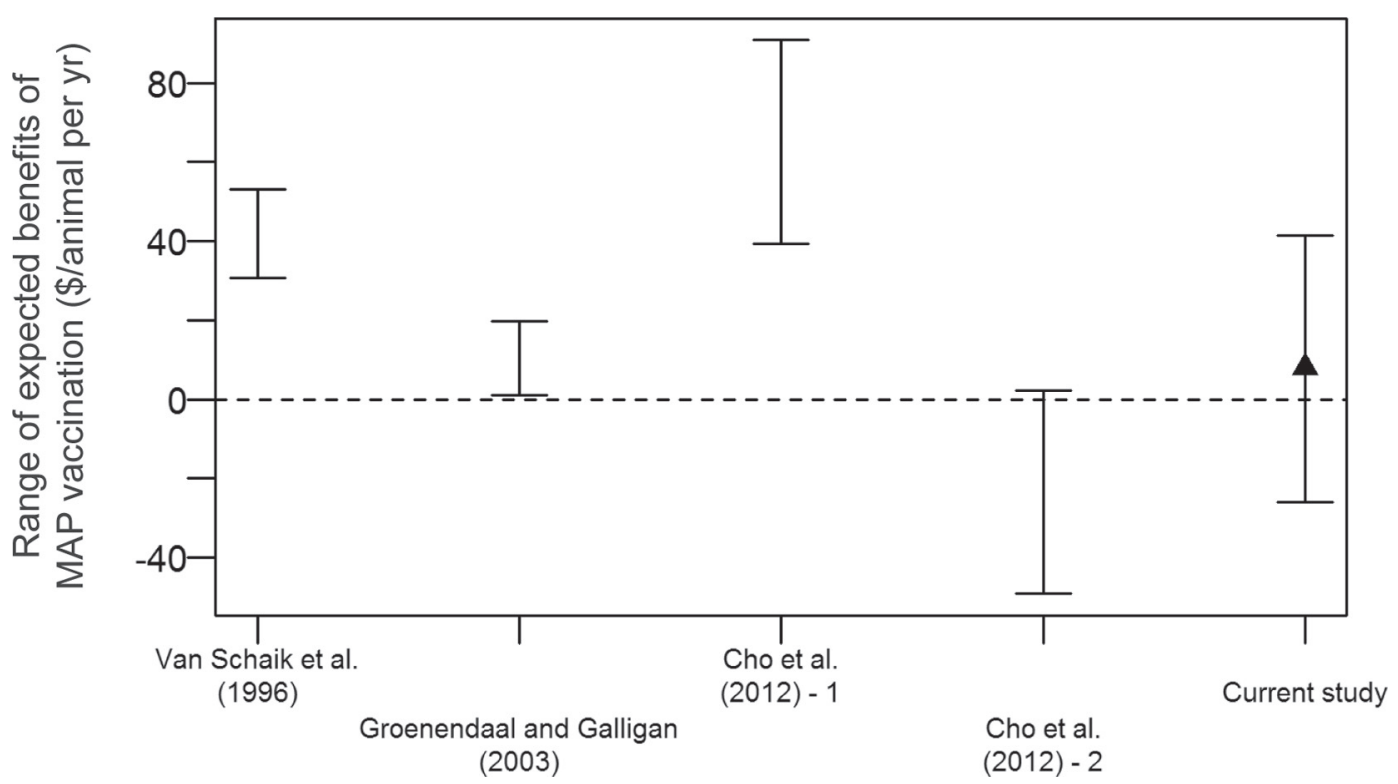

Figure 6. Comparison of the estimates of the direct benefits of Mycobacterium avium ssp. paratuberculosis (MAP) vaccination per animal per year between different studies. Vaccine efficacy from Cho et al. (2012) and from Groenendaal and Galligan (2003) were hypothetical and the lower and upper ends of the ranges indicate benefits with a low- and high-efficacy hypothetical vaccine, respectively. Vaccine efficacy in van Schaik et al. (1996) and the current study were based on field data. The lower and upper ends of the range of van Schaik et al. (1996) indicate a $5 \%$ and $11 \%$ prevalence of Johne's disease clinical cases, respectively. The lower and upper end of the range of the current study indicates the 10th and 90th percentiles (P10 and P90) of the predictive interval (PI) around the triangular, which are the expected direct benefits.

in the current study, controls and vaccinated animals were subject to the same management and therefore the difference between the 2 groups was the net effect of vaccination. In another study, Groenendaal and Galligan (2003) estimated that the net direct benefits range from $\$ 1.07$ to $\$ 20.28$ (average of $\$ 10.34$ ) per animal per year, depending on the assumed (hypothetical) vaccine efficacy (2003 prices inflated to 2014). Their estimates seem consistent with the current results, as the latter are likely an underestimate of the true direct MAP vaccination benefits. In a third study, Cho et al. (2012) found the maximum costs per vaccination dose (i.e., the reduction of losses due to the vaccine) of either a low efficacy or high efficacy vaccine for $10 \%$ prevalence herds to be $\$ 103$ or $\$ 223$, respectively, compared with no control measure being implemented, and $-\$ 102$ and $\$ 17$, respectively, when vaccination was compared with an improved hygiene and test-and-cull strategy. Assuming 43 vaccinated heifer calves per 100 cows per year and a cost of the vaccine of $\$ 12$, this suggests direct annual benefits of MAP vaccination per animal per year of $\$ 39$ and $\$ 91$ without other control measures implemented and $-\$ 49$ and $\$ 2$ when vaccination was compared with an improved hygiene and test-and-cull strategy. Focusing on losses due to JD only (i.e., not on the effects of vaccination on JD losses), Ott et al. (1999) standardized the losses per animal and year due to JD from 6 studies and estimated them to range from $\$ 31$ to $\$ 42$, whereas Groenendaal and Galligan (2003) estimated losses at $\$ 48$ (both expressed in 2014 prices). Given that (1) vaccines do not prevent all JD losses, (2) it takes a few years before benefits start, and (3) there are costs of MAP vaccination, we believe that the true expected net direct annual benefits of MAP vaccination are most likely less than $\$ 25$ to $\$ 30$ per animal, depending on the initial herd prevalence. Given these previous studies, given that the currently calculated benefits of $\$ 8.02$ from the current study are likely underestimated, and given the sensitivity analysis around the reduction of infection probability, the realistic range around the true expected direct benefits of MAP vaccination probably lies in the range of $\$ 10$ to $\$ 25$ per animal per year.

The current study shows that the direct benefits of MAP vaccination increase only slightly with a higher MAP prevalence at the start of the vaccination, which is consistent with Cho et al. (2012). For example (Figure 3 ), there is only a $56 \%$ increase of economic benefits of vaccination when increasing the starting prevalence by $500 \%$ (i.e., from 5 to $25 \%$ ). This is because both in the current study and in Cho et al. (2012) without vaccination, the prevalence is assumed to increase over time and therefore the overall expected economic benefits of vaccination are not directly proportional to the starting prevalence. 


\section{Indirect Effects of MAP Vaccination}

Our overall cost-benefit analysis shows that for a typical US dairy herd (e.g., a MAP-infected herd that is not located within a region where there is a high probability of CFT testing because of, for example, tuberculosis infections), the indirect costs of MAP vaccination are smaller than the direct benefits of vaccination, and therefore MAP vaccination is economically attractive. All direct benefits of MAP vaccination accrue to producers, whereas the majority of the indirect costs are paid for by the government or taxpayers.

\section{Cost-Benefit Break-Even Analysis}

The aim of cost-benefit break-even analysis was to show under what circumstances MAP vaccination is economically attractive or not, considering both direct and indirect costs and benefits. Seven main inputs were varied to assess their effect on the economically preferred strategy.

In general, when varying the main inputs of the costbenefit analysis, vaccination still has greater direct benefits than indirect costs. There are, however, a few exceptions to this.

As shown in Figure 5A, for regions where a high percentage of all animals are CFT tested each year (e.g., $34.52 \%$ in Arizona in 2007), vaccination is not economically attractive. Herd-wide CFT testing is typically due to the presence of local $M$. bovis infections and therefore vaccination is economically not attractive in regions or states that have a high likelihood of tuberculosis infections. As shown in Figure $5 \mathrm{~F}$, if the number of animals CFT tested/herd is 11 or lower and MAP vaccination direct benefits are $\$ 8.03$, vaccination becomes economically unattractive. Our current study assumed by default that animals are CFT tested within large groups (i.e., whole-herd tests), but in situations or states where this is typically not the case the indirect costs of MAP vaccination increase and therefore MAP vaccination overall becomes less attractive. Therefore, we believe that, in most circumstances, the expected total economic benefits of MAP vaccination outweigh the indirect expected economic costs.

All break-even analyses were presented from an overall economic perspective (i.e., combining all costs and benefits together). All direct benefits from MAP vaccination accrue to producers while the majority of the indirect costs are incurred by both producers and the government. From a government or taxpayer perspective, MAP vaccination is not attractive as it always only causes additional costs. When considering only the producers' perspective and ignoring indirect costs at the government level, the expected indirect costs of MAP vaccination are reduced considerably and therefore MAP vaccination becomes economically more attractive. In addition, as calculated in a break-even analysis that considers only the producer-level direct benefits and indirect costs of MAP vaccination (results not shown), MAP vaccination continues to be economically attractive under a wider variety of scenarios, including scenarios with CFT testing of smaller groups of animals. However, this does not mean that for every individual producer, actual direct MAP vaccination benefits are higher than actual indirect costs.

Dressler et al. (2010) also studied the cross-reactivity of MAP vaccination while considering the indirect costs to the BTB program. However, they used $\$ 53.03$ per animal per year as the direct benefits of MAP vaccination, which was based on van Schaik et al. (1996), a study that considered herds with a very high JD prevalence (11\% animals with clinical JD at start of vaccination). Moreover, Dressler et al.'s (2010) perspective was of a dairy herd in a geographic region given a BTB outbreak attributed to surrounding endemically infected wild deer population. Their estimate of the indirect costs of MAP vaccination due to cross-reactivity ranged from $\$ 30.78$ to $\$ 32.72$ per cow per year, which is considerably higher than the $\$ 2.14$ per animal per year estimated in the current study. This difference was mainly due to their assumption of a tuberculosis outbreak in the region. In this study, in contrast, given the very low prevalence of BTB within the United States, the default probability of a random bovine being CFT tested per year (because of, for example, a tuberculosis outbreak in the region) was assumed to be $2.87 \%$ (USDA-APHIS-VS, 2009).

In the current study, the default situation was CFT testing at the herd level. However, as shown in Figures 4 and $5 \mathrm{~F}$, when the number of animals that are CFT tested per testing round is smaller, the marginal indirect costs of MAP vaccination per animal and year due to cross-reactivity increase considerably. The main reason for this is that if individual animals or smaller groups of animals are CFT tested, there will be more instances (i.e., a higher probability) where cross-reactivity causes the first CFT- or CCT-positive animal, which results in costs due to a standstill on the farm at the producer level and additional testing costs at the government level.

This current study assumed an interference between MAP vaccination and BTB testing, as supported by several studies (Köhler et al., 2001; Lima et al., 2012; Thomsen et al., 2012). Although other studies reported no cross-reactivity (Coad et al., 2013; Tewari et al., 2014), they were based on other vaccines or types of BTB tests (i.e., serological tests vs. cell-based tests). In addition, the current study only took into account ad- 
ditional false-positive test results (i.e., cross-reactivity) of MAP vaccinates but did not consider potential additional positive results from MAP-infected animals. Field observations (E. A. Patton) suggest there may be some cross-reactivity of MAP infection with CFT tests, but Lima et al. (2012) found no significant differences in the CFT response rate between MAP-infected and noninfected animals. If MAP infection actually caused cross-reactivity with either the CFT or CCT tests, the indirect costs of vaccination would be less than presented in this study because vaccination does decrease the MAP prevalence.

\section{CONCLUSIONS}

First, the benefits of MAP vaccination were estimated to be positive, at $\$ 8.03$ per animal per year, on average. This likely represents an underestimation of the true direct benefits of MAP vaccination in MAP-infected herds. Second, in most circumstances, the expected direct benefits of MAP vaccination were higher than the expected indirect costs of vaccination due to crossreactivity within the BTB program, resulting in positive total net benefits. Third, as shown in an extensive break-even analysis, for producers in a region or state with a high probability of CFT testing, the indirect costs will increase and may exceed the direct benefits of MAP vaccination. An important consideration with the current conclusions is that all net direct benefits are incurred by producers, whereas the indirect costs are mostly paid for by the government or taxpayer. Overall, our study indicated that vaccination against JD is an economically attractive tool on MAP-infected dairy herds in regions with limited CFT testing. Vaccination for MAP would become even more economically attractive if MAP vaccines or BTB serological tests that minimize cross-reactivity were to become available.

\section{ACKNOWLEDGMENTS}

The authors gratefully acknowledge funding provided by USDA-CSREES award number 2008-37610-19540.

\section{REFERENCES}

Brealey, R. A., and S. C. Myers. 2007. How to calculate present values. Pages 34-55 in Principles of Corporate Finance. 9th ed. McGrawHill, New York, NY.

Briggs, A. H., M. C. Weinstein, E. A. Fenwick, J. Karnon, M. J. Sculpher, and A. D. Paltiel. 2012. Model parameter estimation and uncertainty analysis: A report of the ISPOR-SMDM Modeling good research practices task force working group-6. Med. Decis. Making 32:722-732.

Cho, J., L. W. Tauer, Y. H. Schukken, M. I. Gomez, R. L. Smith, Z. Lu, and Y. T. Grohn. 2012. Economic analysis of Mycobacterium avium subspecies paratuberculosis vaccines in dairy herds. J. Dairy Sci. 95:1855-1872.

Coad, M., D. J. Clifford, H. M. Vordermeier, and A. O. Whelan. 2013. The consequences of vaccination with the Johne's disease vaccine, Gudair, on diagnosis of bovine tuberculosis. Vet. Rec. 172:266.

Collins, M. T., V. Eggleston, and E. J. Manning. 2010. Successful control of Johne's disease in nine dairy herds: results of a six-year field trial. J. Dairy Sci. 93:1638-1643.

Dorshorst, N. C., M. T. Collins, and J. E. Lombard. 2006. Decision analysis model for paratuberculosis control in commercial dairy herds. Prev. Vet. Med. 75:92-122.

Dressler, J. B., R. L. Smith, L. W. Tauer, Y. H. Schukken, and Y. T. Grohn. 2010. Economic analysis of the cross-reactivity of Johne's Disease vaccination with tuberculosis in dairy cattle. Am. J. Agric. Econ. 92:1446-1455.

Espejo, L., F. J. Zagmutt, H. Groenendaal, C. Munoz-Zanzi, and S. J. Wells. 2015. Evaluation of performance of bacterial culture of feces and serum ELISA across stages of Johne's disease in cattle using a Bayesian latent class model. J. Dairy Sci. http://dx.doi. org/10.31.68/jds.2014-8440.

Farnham, M. W., B. Norby, T. J. Goldsmith, and S. J. Wells. 2012. Meta-analysis of field studies on bovine tuberculosis skin tests in United States cattle herds. Prev. Vet. Med. 103:234-242.

Groenendaal, H., and D. T. Galligan. 2003. Economic consequences of control programs for paratuberculosis in midsize dairy farms in the United States. J. Am. Vet. Med. Assoc. 223:1757-1763.

Groenendaal, H., D. T. Galligan, and H. A. Mulder. 2004. An economic spreadsheet model to determine optimal breeding and replacement decisions for dairy cattle. J. Dairy Sci. 87:2146-2157.

Groenendaal, H., M. Nielen, and J. W. Hesselink. 2003. Development of the Dutch Johne's disease control program supported by a simulation model. Prev. Vet. Med. 60:69-90.

Groenendaal, H., and C. A. Wolf. 2008. Farm-level economic analysis of the US national Johne's Disease demonstration herd project. J. Am. Vet. Med. Assoc. 233:1852-1858.

Knust, B., D. Konkle, J. J. Bohn, S. J. Wells, and E. Patton. 2009. Johne's disease vaccine: A cohort study measuring long-term effectiveness of the whole cell killed bacterin. Page 235 in Proc. 10th Int. Colloq. Paratuberculosis, Minneapolis, MN. University of Minnesota, Minneapolis.

Knust, B., E. Patton, J. Ribeiro-Lima, J. J. Bohn, and S. J. Wells 2013. Evaluation of the effects of a killed whole-cell vaccine against Mycobacterium avium subsp paratuberculosis in 3 herds of dairy cattle with natural exposure to the organism. J. Am. Vet. Med. Assoc. 242:663-669.

Köhler, H., H. Gyra, K. Zimmer, K. G. Drager, B. Burkert, B. Lemser, D. Hausleithner, K. Cubler, W. Klawonn, and R. G. Hess. 2001. Immune reactions in cattle after immunization with a Mycobacterium paratuberculosis vaccine and implications for the diagnosis of M. paratuberculosis and M. bovis infections. J. Vet. Med. B Infect. Dis. Vet. Public Health 48:185-195.

Kudahl, A. B., S. Ostergaard, J. T. Sorensen, and S. S. Nielsen. 2007. A stochastic model simulating paratuberculosis in a dairy herd. Prev. Vet. Med. 78:97-117.

Larsen, A. B., A. I. Moyle, and E. M. Himes. 1978. Experimental vaccination of cattle against paratuberculosis (Johne's disease) with killed bacterial vaccines: a controlled field study. Am. J. Vet. Res. 39:65-69

Lima, R. J., E. Patton, L. Glazer, B. Carlson, and S. J. Wells. 2012. Impact of Johne's disease, natural infection and vaccination, on bovine tuberculosis diagnostics tests. Presentation 042P in Proc. 93rd Annu. Meet. Conf. Res. Workers Anim. Dis. (CRWAD), Chicago, IL. Coe-Truman Technologies, Inc., Chicago, IL.

Lombard, J. E., I. A. Gardner, S. R. Jafarzadeh, C. P. Fossler, B. Harris, R. T. Capsel, B. A. Wagner, and W. O. Johnson. 2013. Herd-level prevalence of Mycobacterium avium ssp. paratuberculosis infection in United States dairy herds in 2007. Prev. Vet. Med. 108:234-238.

Lu, Z., Y. H. Schukken, R. L. Smith, R. M. Mitchell, and Y. T. Grohn. 2013. Impact of imperfect Mycobacterium avium ssp. paratubercu- 
losis vaccines in dairy herds: A mathematical modeling approach. Prev. Vet. Med. 108:148-158.

National Agricultural Statistics Service (NASS). 2014. National Statistics for Milk. Accessed Jan. 15, 2015. http://www.nass.usda. gov/Statistics_by_Subject/result.php?6D0E732E-DB29-3FE59D2B-C130F5E1551F\&sector $=$ ANIMALS $\% 20 \% 26 \% 20$ PRODUC TS\&group=DAIRY\&comm $=$ MILK.

National Research Council (NRC) Committee on Diagnosis and Control of Johne's Disease. 2003. Johne's Disease and Crohn's Disease. Pages 104-120 in Diagnosis and Control of Johne's Disease. National Academies Press, Washington, DC.

Ott, S. L., S. J. Wells, and B. A. Wagner. 1999. Herd-level economic losses associated with Johne's disease on US dairy operations. Prev. Vet. Med. 40:179-192.

Radia, D., K. Bond, G. Limon, S. van Winden, and J. Guitian. 2013. Relationship between periparturient management, prevalence of MAP and preventable economic losses in UK dairy herds. Vet. Rec. 173:343.

Raizman, E. A., S. J. Wells, C. A. Muñoz-Zanzi, and S. Tavornpanich. 2011. Estimated within-herd prevalence (WHP) of Mycobacterium avium ssp. paratuberculosis in a sample of Minnesota dairy herds using bacterial culture of pooled fecal samples. Can. J. Vet. Res. 75:112-116.

Tewari, D., E. Hovingh, R. Linscott, E. Martel, J. Lawrence, D. Wolfgang, and D. Griswold. 2014. Mycobacterium avium ssp. paratuberculosis antibody response, fecal shedding, and antibody crossreactivity to Mycobacterium bovis in M. avium ssp. paratuberculosis-infected cattle herds vaccinated against Johne's disease. Clin. Vaccine Immunol. 21:698-703.
Thomsen, V. T., S. S. Nielsen, A. Thakur, and G. Jungersen. 2012 Characterization of the long-term immune response to vaccination against Mycobacterium avium ssp. paratuberculosis in Danish dairy cows. Vet. Immunol. Immunopathol. 145:316-322.

USAHA. 2004. Report of the Committee on Tuberculosis. Accessed Jan. 15, 2015. http://portals5.gomembers.com/portals/6/ reports/2004/report-tb-2004.pdf.

USDA-APHIS. 2004. Bovine tuberculosis eradication-Uniform methods and rules, Effective January 1, 2005. 1-29. APHIS 91-45011. U.S. Government Printing Office, Washington, DC. Accessed Jun. 8, 2015. http://www.aphis.usda.gov/animal_health/animal_ diseases/tuberculosis/downloads/tb-umr.pdf.

USDA-APHIS-VS. 2009. Analysis of bovine tuberculosis surveillance in accredited free states. Accessed Jan. 15, 2015. http://www aphis.usda.gov/vs/nahss/cattle/tb_2009_evaluation_of_tb_in_ accredited_free_states_jan_09.pdf.

van Schaik, G., C. H. Kalis, G. Benedictus, A. A. Dijkhuizen, and R. B. Huirne. 1996. Cost-benefit analysis of vaccination against paratuberculosis in dairy cattle. Vet. Rec. 139:624-627.

Zagmutt, F. J., L. Espejo, H. Groenendaal, R. J. Lima, E. Patton, and S. J. Wells. 2012. A simulation method to reconstruct the longitudinal animal and herd level infection status based on repeated screening tests with no gold standard: Application to paratuberculosis modeling. Page 53 in Proc. 13th Int. Symp. Vet. Epidemiol Econ., Maastricht, the Netherlands. Wageningen Academic Publishers, Wageningen, the Netherlands. 